\title{
Enhanced thermoelectric performance of skutterudites via orthogonal experimental design
}

\author{
L. Deng ${ }^{a *}$, J M. Qin ${ }^{\mathrm{a}}$, X P. Jia ${ }^{\mathrm{b}}$, X. Guo ${ }^{\mathrm{a}}$. \\ ${ }^{a}$ Department of Material Science and Engineering, Changchun University of \\ Science and Technology, Changchun 130022, China; \\ ${ }^{\mathrm{b}}$ National Lab of Superhard Materials, Jinlin University, Changchun 130012, China
}

\begin{abstract}
A novel chemical alloying method of high-pressure and high-temperature (HPHT) has been used for the synthesis of bulk-skutterudite Te-doped $\mathrm{CoSb}_{3}$. Because the thermoelectric properties of skutterudites could be effectively optimized by changing synthetic pressure and temperature, and doping concentration, the orthogonal experimental design method has been applied. The Seebeck coefficient, electrical resistivity and thermal conductivity of $\mathrm{CoSb}_{3}$ were all performed in the temperature range of $371-710 \mathrm{~K}$. The figure of merit, ZTs, increase with the increasing synthesis pressures until $2.3 \mathrm{GPa}$, and thereafter they decrease; the thermal conductivity decrease with the increasing synthesis pressures until 2.3GPa, and thereafter they increase. At last, the maximum ZT value of sample $\mathrm{Co}_{4} \mathrm{Sb}_{11.5} \mathrm{Te}_{0.5}$ prepared at $2.3 \mathrm{GPa}$ reached 1.03 at $710 \mathrm{~K}$ due to its lower thermal conductivity and higher power factor.
\end{abstract}

Keywords: Skutterudite; Thermoelectric properties; HPHT; Seebeck coefficient

\section{Introduction}

Thermoelectric materials have attracted much attention in these years, which can directly convert heat into electricity in a safe and clean way. As stated earlier, skutterudite compounds have been extensively studied as a group of promising thermoelectric materials over the last decade. Interest in these materials was greatly increased by the introduction of the phonon glass electron crystal (PGEC) concept [1-3]. The performance of a thermoelectric material is generally characterized by the dimensionless figure of merit, $\mathrm{ZT}=\alpha^{2} \sigma \mathrm{T} / \kappa[4,5]$, where $\alpha, \sigma, \mathrm{T}$ and $\kappa$ are the Seebeck coefficient, electrical conductivity, absolute temperature, and total thermal conductivity, respectively. Generally, $\kappa$ is sum of the electronic thermal conductivity $\kappa_{\mathrm{e}}$ and the lattice thermal conductivity $\kappa_{\mathrm{ph}}$.

Because the research of $\mathrm{CoSb}_{3}$ has been greatly developed, it is necessary to further research on $\mathrm{CoSb}_{3}$ in order to meet the requirement of commercial production. So far, there has been no research that shows the upper limit of ZT, current research results have maximum ZT $=1 \sim 2$ due to adversely inter-related $\alpha, \sigma$, and $\kappa$ where optimizing one quantity often unfavorably affects the others. There are also several studies for the substitution of Sb for instance by Pd, Te or Sn. The main goal of the doping $[6,7]$ is to achieve a large power factor or to prepare p-type and n-type $\mathrm{CoSb}_{3}$ required for some modules.

In addition to chemical substitution, pressure tuning is deemed to be an alternative strategy, but in situ measurements on as-synthesized samples show severe reversibility. [8,9] However, the HPHT technique is effective not only for synthesizing pure-phase bulks directly from elements in a short time, but also for tuning their thermoelectric properties simultaneously. [10,11] Besides, the synthesis time is sharply reduced to about half an hour, which is advantageous for scalable production. Since synthesis pressure, synthesis temperature and doping concentration all have significant effects on the thermoelectric properties of $\mathrm{CoSb}_{3}$ skutterudites, the orthogonal test design has been taken to obtain the

${ }^{*}$ To whom correspondence should be addressed. Fax, 86-431-85583016, Email: dengyue520619@126.com. 
best ZT values sample. First, samples $\mathrm{Co}_{4} \mathrm{Sb}_{12-\mathrm{x}} \mathrm{Te}_{\mathrm{x}}$ of different doping concentrations were prepared, and then the doping concentration of the best thermoelectric properties samples was selected to synthesis at different pressures. Finally, the maximum ZT value of sample $\mathrm{Co}_{4} \mathrm{Sb}_{11.5} \mathrm{Te}_{0.5}$ prepared at $2.3 \mathrm{GPa}$ reached 1.03 at $710 \mathrm{~K}$. This method not only reduced the number of experiments, but also broadened the optimization approach of thermoelectric materials.

\section{Experimental procedure}

\subsection{Sample preparation}

The $\mathrm{Co}_{4} \mathrm{Sb}_{12-\mathrm{x}} \mathrm{Te}_{\mathrm{x}}$ samples were prepared with $\mathrm{Te}, \mathrm{Sb}, \mathrm{Co}$, (99.9\% in purity) powders as sources, which were weighed according to the stoichiometry. After being uniformly mixed in an agate mortar under argon protection, the mixtures were shaped by pressing. The mixtures were shaped to a cylinder with about $3 \mathrm{~mm}$ thick and $10 \mathrm{~mm}$ in diameter by press. The cylinder samples were assembled for HPHT synthesis. The samples were prepared in a cubic anvil high pressure apparatus (SPD $6 \times 1200$ ) with a sample chamber of $23 \mathrm{~mm}$ on an edge at $900 \mathrm{~K}$ and 1.5-3.7GPa. Samples were heated 25 minutes under the corresponding synthesis pressure, and then stopped heating immediately. After unloading the pressure to the atmospheric pressure, the samples were polished and tested.

\subsection{Sample characterization}

$\mathrm{X}$-ray diffraction (XRD) measurements with $\mathrm{Cu}-\mathrm{K}_{\alpha}$ radiation were performed on an X-ray diffractometer (D/MAX-RA). The morphology and microstructure of synthetic $\mathrm{Co}_{4} \mathrm{Sb}_{12-\mathrm{x}} \mathrm{Te}_{\mathrm{x}}$ samples were examined using field emission scanning electron microscope (SEM, Jeol JSM-6700F) and a high-resolution transmission electron microscopy (HRTEM, Jeol JEM-2100F). The Seebeck coefficient and electrical conductivity were measured simultaneously by a ZEM-3 apparatus, and which were measured in the temperature range from $371 \mathrm{~K}$ to $710 \mathrm{~K}$. The thermal conductivity $\kappa$ was measured on a TC-7000 (ULVAC-RIKO Inc., Japan) Laser Flash Thermal Constants Measuring Apparatus which were measured in the temperature range of $371-710 \mathrm{~K}$.

\section{Results and discussion}

\subsection{XRD and FESEM}

Typical XRD patterns of $\mathrm{Co}_{4} \mathrm{Sb}_{11.5} \mathrm{Te}_{0.5}$ samples are shown in Fig. 1. (a) The XRD patterns of $\mathrm{Co}_{4} \mathrm{Sb}_{11.5} \mathrm{Te}_{0.5}$ samples prepared under different synthetic pressure are nearly the same. It can be known that the major phases of $\mathrm{Co}_{4} \mathrm{Sb}_{11.5} \mathrm{Te}_{0.5}$ are all matched to that of the single phase of $\mathrm{CoSb}_{3}$ at normal pressure, which indicates that the samples prepared by HPHT are nearly with the single phase $\mathrm{CoSb}_{3}$. In addition, compared with the traditional method, the processing time of HPHT method has been reduced from a few days to half an hour.

Fig. 1. (b) and (c) are the SEM micrographs of $\mathrm{Co}_{4} \mathrm{Sb}_{11.5} \mathrm{Te}_{0.5}$, which were prepared under $1.5 \mathrm{GPa}$ and $2.3 \mathrm{GPa}$, respectively. From Fig. 1. (b) it can be seen that the mean grain size is about $0.5-2 \mu \mathrm{m}$, but Fig. 1. (c) has a smaller grain size and more abundant grain boundaries than the former. The advantages of the HPHT technique include the ability to tune rapidly, restraint of disorder and phase separation, introduction of more abundant grain boundary, and so on. The SEM micrographs of the samples prove the advantage of HPHT synthesis of introducing an abundant grain boundary. 

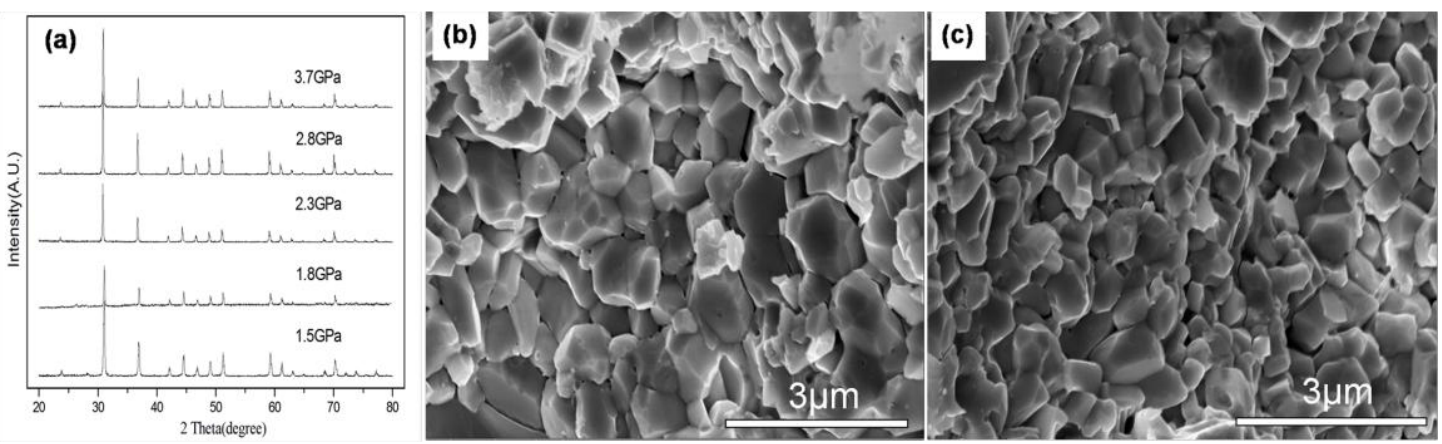

Fig. 1. (a) XRD patterns of $\mathrm{Co}_{4} \mathrm{Sb}_{11.5} \mathrm{Te}_{0.5}$ bulks by various pressures. FESEM images of fractured surfaces of $\mathrm{Co}_{4} \mathrm{Sb}_{11.5} \mathrm{Te}_{0.5}$ prepared at (b) $1.5 \mathrm{GPa}$, (c) $2.3 \mathrm{GPa}$.

\subsection{TEM and HRTEM}

Fig. 2. (a) shows numerous distortions and dislocations embedded in the matrix. A certain amount of localized wavy patterns inside of all the crystal grains have been found, such as the white circle area. In Fig. 2. (b), the strips which were circled by white line in Fig. 2. (a) were performed by HRTEM. These nanostructures are coherent with their surrounding crystal matrix, the spacing of the stripes is about $1-3 \mathrm{~nm}$. Similarly, the strip features are similar to those in previous works [12, 13], which is called structural modulations. But for $\mathrm{Co}_{4} \mathrm{Sb}_{11.5} \mathrm{Te}_{0.5}$ sample, the distribution of strip structure is much more. Fig. 2. (b) shows many kinds of disorder, such as lattice curvatures and dislocations. These curvatures and dislocations are enlarged and displayed in the small map of Fig. 2. (b). The occurrence of the disorder is mainly due to the preparation method of high pressure quenching, and higher synthesis pressure can produce more dislocations

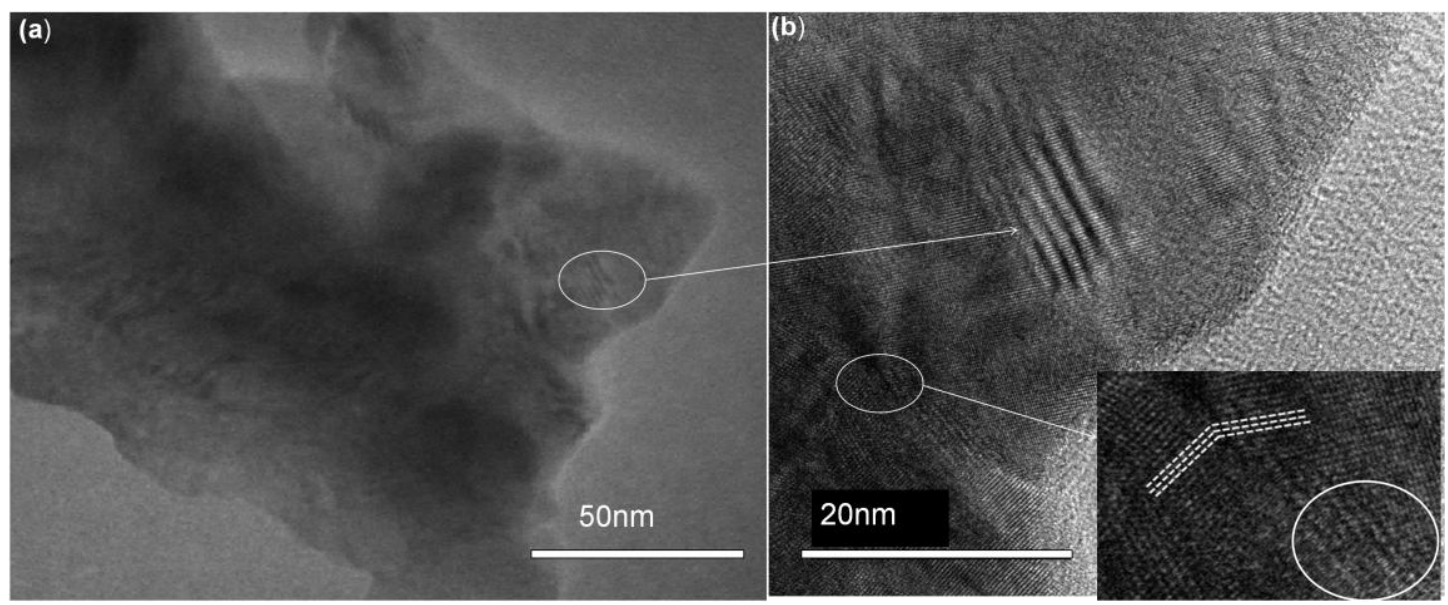

Fig. 2. (a) TEM image and (b) HRTEM image of $\mathrm{Co}_{4} \mathrm{Sb}_{11.5} \mathrm{Te}_{0.5}$ sample prepared at $2.3 \mathrm{GPa}$.

\subsection{Thermoelectric properties}

Figure. 3. (a) shows the synthesis pressure dependence of the electrical resistivity for $\mathrm{Co}_{4} \mathrm{Sb}_{11.5} \mathrm{Te}_{0.5}$ samples at different temperature. The electrical resistivity of the samples increases with the increasing synthesis pressure in temperature range of 371-710 $\mathrm{K}$ except the sample prepared at 1.8GPa. It can be noted that all the compounds possess small values of electrical resistivity at $371 \mathrm{~K}$, and the minimum value of $0.92 \mathrm{~m} \Omega \mathrm{cm}$ for $\mathrm{Co}_{4} \mathrm{Sb}_{11.5} \mathrm{Te}_{0.5}$ is obtained at $1.8 \mathrm{GPa}$.

Figure. 3. (b) shows the synthesis pressure dependences of Seebeck coefficient for $\mathrm{Co}_{4} \mathrm{Sb}_{11.5} \mathrm{Te}_{0.5}$ samples at different temperature. All samples exhibit $n$-type conduction as they have negative Seebeck 
coefficient. Samples with different synthesized pressure show a similar $T$ dependence trend for the Seebeck coefficient from $371 \mathrm{~K}$ to $662 \mathrm{~K}$, with the maximum absolute value of Seebeck coefficient occurring at about $662 \mathrm{~K}$ for $\mathrm{Co}_{4} \mathrm{Sb}_{11.5} \mathrm{Te}_{0.5}$ samples synthesized at 2.3-3.7GPa. The absolute value of Seebeck coefficient of the sample synthesized at $2.3 \mathrm{GPa}$ gradually increases with increasing temperature until $662 \mathrm{~K}$, and thereafter it decreases slightly. Its maximum absolute value attains to $227.5 \mu \mathrm{VK}^{-1}$ 。

Power factor (PF) was calculated from the measured Seebeck coefficient and electrical resistivity as shown in Fig. 3. (c). The mini figure in Fig. 3. (c) is $\mathrm{Co}_{4} \mathrm{Sb}_{12-\mathrm{x}} \mathrm{Te}_{\mathrm{x}}$ prepared at $1.5 \mathrm{GPa}$, it can be seen that $\mathrm{Co}_{4} \mathrm{Sb}_{11.5} \mathrm{Te}_{0.5}$ shows the largest PF values both at room temperature and high temperature. So, $\mathrm{Co}_{4} \mathrm{Sb}_{11.5} \mathrm{Te}_{0.5}$ sample has the space to be further optimized by HPHT. The power factor $\mathrm{PF}=\alpha^{2} / \rho[14-16]$ of the samples $\mathrm{Co}_{4} \mathrm{Sb}_{11.5} \mathrm{Te}_{0.5}$ prepared at different pressures increase rapidly with an increase of the temperature $\mathrm{T}$, mainly due to the increase of Seebeck coefficient with increasing T. At $710 \mathrm{~K}$, all $\mathrm{Co}_{4} \mathrm{Sb}_{11.5} \mathrm{Te}_{0.5}$ samples prepared at different pressures show the largest PF values. The largest PF values are between $30-36 \mu \mathrm{w} / \mathrm{cmK}^{2}$.
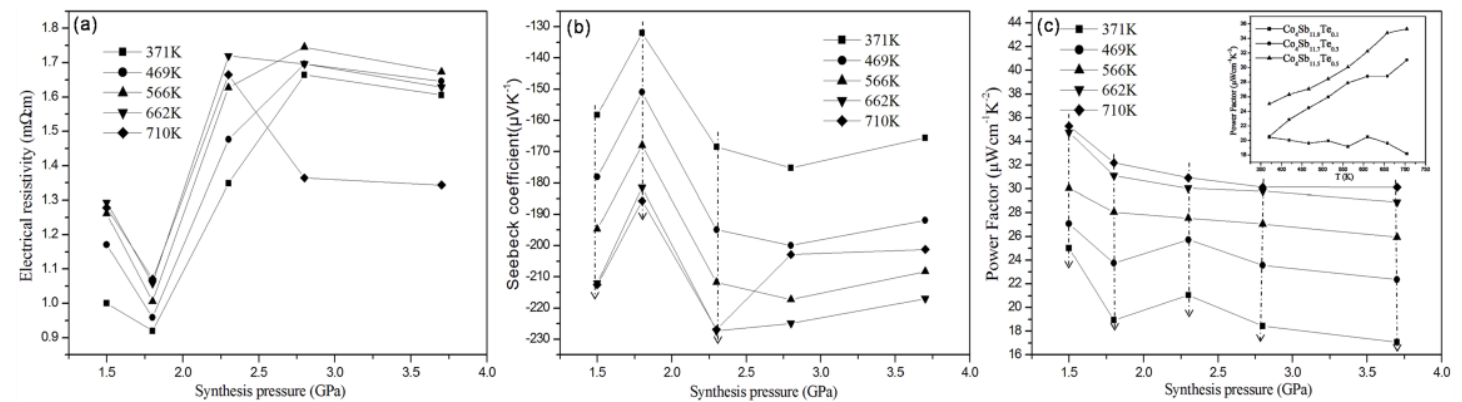

Fig. 3. (a) presents the synthesis pressure dependences of (a) electrical resistivity,(b) Seebeck coefficient and (c) Power factor of $\mathrm{Co}_{4} \mathrm{Sb}_{11.5} \mathrm{Te}_{0.5}$ samples at different temperature, the mini figure in (c) is $\mathrm{Co}_{4} \mathrm{Sb}_{12-\mathrm{x}} \mathrm{Te}_{\mathrm{x}}$ prepared at $1.5 \mathrm{GPa}$.

The synthesis pressure dependences of thermal conductivity are plotted versus temperature in Fig. 4. (a). It can be seen that the thermal conductivities decrease with the increasing temperature for sample prepared at 1.5 and $2.3 \mathrm{GPa}$. The thermal conductivities of sample prepared at $1.8 \mathrm{GPa}$ are not affected by temperature. The thermal conductivities of sample prepared at 2.3GPa are lower than other samples except at $371 \mathrm{~K}$. The minimum value of $\mathrm{Co}_{4} \mathrm{Sb}_{11.5} \mathrm{Te}_{0.5}$ synthesized at $2.3 \mathrm{GPa}$ is $2.13 \mathrm{Wm}^{-1} \mathrm{~K}^{-1}$, which is about 1 times lower than that of sample $\mathrm{Co}_{4} \mathrm{Sb}_{11.5} \mathrm{Te}_{0.5}$ prepared at $1.5 \mathrm{GPa}$. The reduction in thermal conductivity is mainly because of the decreased $\kappa_{\mathrm{ph}}$, which was caused by the enhancement of point defect scattering and the disorder among the lattices. The lattice thermal conductivity $\kappa_{\mathrm{ph}}$ is shown in Fig. 4. (b). Lattice thermal conductivity $\kappa_{\mathrm{ph}}$ is calculated by $\kappa_{\mathrm{ph}}=\kappa-\kappa_{\mathrm{e}}$, where $\kappa_{\mathrm{e}}$ is the electronic thermal conductivity, $\kappa_{\mathrm{ph}}$ is lattice component. $\kappa_{\mathrm{e}}$ can be estimated from Wiedemann-Franz's law as $\kappa_{\mathrm{e}}=\mathrm{L} \sigma \mathrm{T}$, where $\mathrm{L}$ is the Lorenz number. Because Te-doped $\mathrm{CoSb}_{3}$ is non degenerate semiconductor, $\mathrm{L}=1.7 \times 10^{-8} \mathrm{~W} \Omega K^{-2}$ is more suitable. The lowest $\kappa_{\mathrm{ph}}$ of $1.34 \mathrm{Wm}^{-1} \mathrm{~K}^{-1}$ has been obtained for the sample prepared at $1.8 \mathrm{GPa}$, which is a little lower than that of sample prepared at 2.3GPa. These results show that HPHT method can restrain the thermal conductivity effectively because of the high pressure can increase the plastic strain of the crystal, then caused an increase of the average dislocation density which increases linearly in a crystal with the plastic strain, and finally 
reduced the $\kappa_{\mathrm{ph}}[17]$.

The synthesis pressure dependences of ZT values are plotted versus temperature in Fig. 4 (c) It can be seen that the ZT values of samples all increase with the increasing temperature. The ZT values of all samples increased with the increase of the synthesis pressure until 2.3GPa, and then decreased. At any temperature, the ZT value of sample $\mathrm{Co}_{4} \mathrm{Sb}_{11.5} \mathrm{Te}_{0.5}$ synthesized at $2.3 \mathrm{GPa}$ are all higher than others, and reaches 1.03 at $710 \mathrm{~K}$, which is mainly attributed to the reduced thermal conductivity. This result shows that the figure of merit for many thermoelectric materials could be improved by appropriate synthesis pressure.
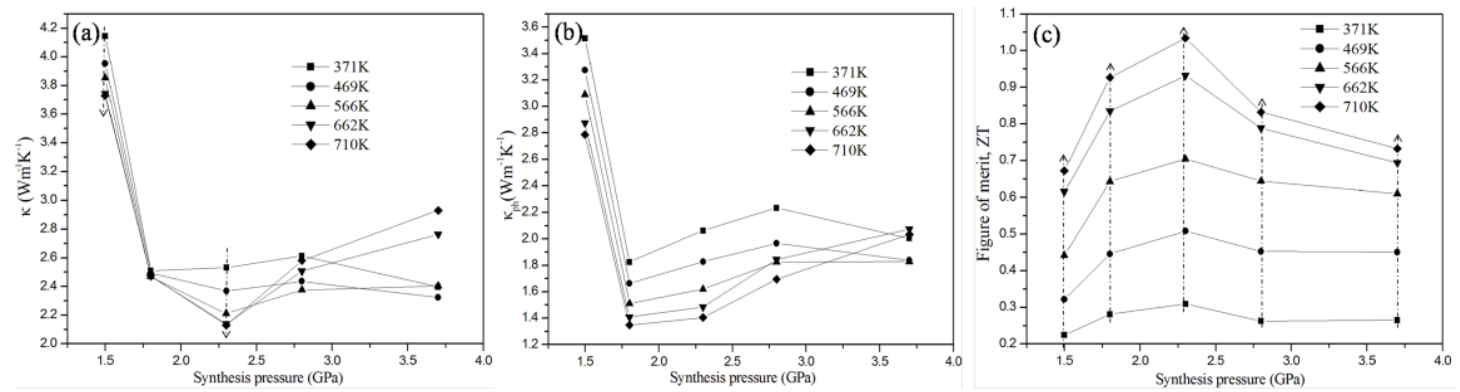

Fig.4. (a) presents the synthesis pressure dependences of (a) thermal conductivity, (b) lattice thermal conductivity $\kappa_{\mathrm{ph}}$ and (c) ZT values at different temperature

\section{Conclusion}

The single phase skutterudite compounds $\mathrm{Co}_{4} \mathrm{Sb}_{11.5} \mathrm{Te}_{0.5}$ were successfully optimized by HPHT. The thermoelectric properties could be improved significantly at high pressure and the excellent properties gained at high pressure have been partially kept to ambient pressure. A minimum value of $\mathrm{Co}_{4} \mathrm{Sb}_{11.5} \mathrm{Te}_{0.5}$ synthesized at $2.3 \mathrm{GPa}$ is $2.13 \mathrm{Wm}^{-1} \mathrm{~K}^{-1}$, and the figure of merit $\mathrm{ZT}$ value takes a maximum 1.03 of $\mathrm{Co}_{4} \mathrm{Sb}_{11.5} \mathrm{Te}_{0.5}$ prepared at 2.3GPa. The experimental results indicated that HPHT method can effectively optimize the series of materials, and maybe $2.3 \mathrm{GPa}$ is a good synthesis pressure.

\section{Acknowledgments}

This work was financially supported by the National Science Foundation of China (51301024 and 51171070) and JiLin Provincial Science and Technology Department Project (20150520026JH).

\section{References}

[1] F.J. Disalvo, Science. 285 (1999) 703-6.

[2] G.S. Nolas, G.A. Slack, D.T. Morelli, T.M. Tritt, A.C. Ehrlich, J. Appl. Phys. 79 (1996) 4002.

[3] T.M. Tritt, G.S. Nolas, G.A. Slack, E A.C. hrlich, J. Appl. Phys. 79 (1996) 8412.

[4] K. Zhang, M.B. Katz, B. Li, S.J. Kim, X. Du, X. Hao , Adv. Mater. 26 (2014) 7365-7370.

[5] D.G. Zhao, M. Zuo, J.F. Leng, H.R. Geng, Intermetallics. 40 (2013) 71-5.

[6] X. H. Li, Q. Zhang, Y. L. Kang, C. Chen, L. Zhang, D. L. Yu, J. Alloys Comp. 677 (2016) 61-65.

[7] J.L. Mi, X.B. Zhao, T.J. Zhu, J. Ma, J. Alloys. Compds. 452 (2008) 225-229.

[8] D. Polvani, J. Meng, N. C. Shekar, J. Sharp, J. Badding, Chem. Mater. 13 (2001) 2068-2071.

[9] J. Meng, N.C. Shekar, J. Badding, D. Chung, M. Kanatzidis, J. Appl. Phys. 90 (2001) 2836-2839.

[10] H. Sun, X. Jia, L. Deng, P. Lv, X. Guo, Y. Zhang, J. Mater. Chem. A. 3 (2015) 4637-4641.

[11] Y. Zhang, X. Jia, L. Deng, X. Guo, H. Sun, B. Sun, J. Alloys Compd. 632 (2015) 514-519.

[12] W.S. Liu, Q. Zhang, Y. Lan, S. Chen, X. Yan, Q. Zhang, Adv. Energy Mater. 1 (2011) 577-578. 
[13] N. Peranio, O. Eibl, J. Appl. Phys. 103 (2008) 024314.

[14] X. Su, H. Li, G. Wang, H. Chi, X. Zhou, X. Tang, Chem. Mater. 23 (2011) 2948.

[15] Y.F. Liu, M.H. Zhou, J. He, Scripta. Materialia. 111 (2016) 39-43

[16] J. Yang, W. Zhang, S. Q. Bai, Z. Mei, L. D. Chen, Appl. Phys. Lett. 90 (2007) 192111.

[17] C. Peter, Phys. Rev. Lett. 2 (1959) 336-338. 